\title{
Primary Umbilical Endometriotic Nodule with an Ultrasound Incidental Finding of Ovarian Endometrioma and a Solitary Intramural Uterine Myoma: A Case Report
}

\author{
Ifeanyi Martin Okoye ${ }^{1^{*}}$, Olufemi Omiwole ${ }^{2}$ and Oluwatoyosi Mary Adeoye \\ ${ }^{1}$ Department of Obstetrics and Gynaecology, Westcare Specialist Hospital, Lagos, Nigeria \\ ${ }^{2}$ Department of Family Medicine, Westcare Specialist Hospital, Lagos, Nigeria \\ ${ }^{3}$ All Saints University School of Medicine, Dominica
}

*Corresponding author: Ifeanyi Martin Okoye, Department of Obstetrics and Gynaecology, Westcare Specialist Hospital, Lagos, Nigeria

\begin{abstract}
Endometriosis is the presence of endometrial stroma outside the uterine cavity. Endometrioma (chocolate cyst) is the most common form of endometriosis. Primary umbilical endometriosis (PUE) is rare, with a prevalence of $<1 \%$ of endometriosis cases. Our case is a 25 -year-old nulliparous female with a three-year history of infertility presenting with primary umbilical endometriosis and an abdomino-pelvic ultrasonography finding of a right endometrioma, and a solitary intramural uterine myoma. These three findings in a patient are a rare occurrence. Abdominopelvic ultrasound and excision biopsy was performed to arrive at a diagnosis.
\end{abstract}

\section{Keywords}

Endometrioma, Primary umbilical endometriotic nodule, Intramural uterine myoma, Ultrasoundscan, MRI, Laparoscopy

\section{Introduction}

Endometriosis is a benign disease characterized by the presence of endometrial tissue outside the uterus $[1,2]$. Endometriosis can occur in nearly every tissue and organ, including lungs, urinary tracts $[3,4]$ and the ovaries [4]. It was first described in 1690 by a German physician Daniel Schroen [1]. Endometriosis affects roughly $10 \%$ (190 million) of reproductive-age women globally [5]. Infertility is a common complication found in four out of ten women who have endometriosis [6]. In addition, endometriosis is implicated in $70 \%$ of chronic pelvic pain in patients with dysmenorrhea [5]. On average, there is a 7 to 10 years delay in diagnosis [7]. Subcutaneous endometriosis describes the types of endometriosis found in the skin and subcutaneous tissues. It accounts for 0.03 to $0.15 \%$ of cases [1]. It presents in two forms: Primary and secondary umbilical endometriosis. Primary umbilical endometriosis, also known as villar's nodules (villar first described the scenario in 1886), arises spontaneously with unclear pathogenetic physiology, and it constitutes $75 \%$ of cases of all umbilical endometriosis. The secondary type results from surgical procedures (laparoscopic and open surgeries) involving the umbilicus [8].

\section{Case Description}

A 25-year-old nulliparous female with a medical history of infertility for three years presented with a complaint of cyclic dull aching umbilical pain that has been present for seven years and does not radiate. The pain worsens during menstrual flow and is relieved with analgesics. The patient also complained of dysmenorrhoea and deep dyspareunia.

On physical examination, the vital signs were normal. A hyperpigmented supraumbilical nodule was noted. The nodule was warm, tender, and measured $2-3 \mathrm{~cm}$ in size (Figure 1).

The rest of the exam was unremarkable. A differential

Citation: Okoye IM, Omiwole O, Adeoye OM (2021) Primary Umbilical Endometriotic Nodule with an Ultrasound Incidental Finding of Ovarian Endometrioma and a Solitary Intramural Uterine Myoma: A Case Report. Obstet Gynecol Cases Rev 8:209. doi.org/10.23937/2377-9004/1410209

Accepted: September 13, 2021: Published: September 15, 2021

Copyright: (c) 2021 Okoye IM, et al. This is an open-access article distributed under the terms of the Creative Commons Attribution License, which permits unrestricted use, distribution, and reproduction in any medium, provided the original author and source are credited. 


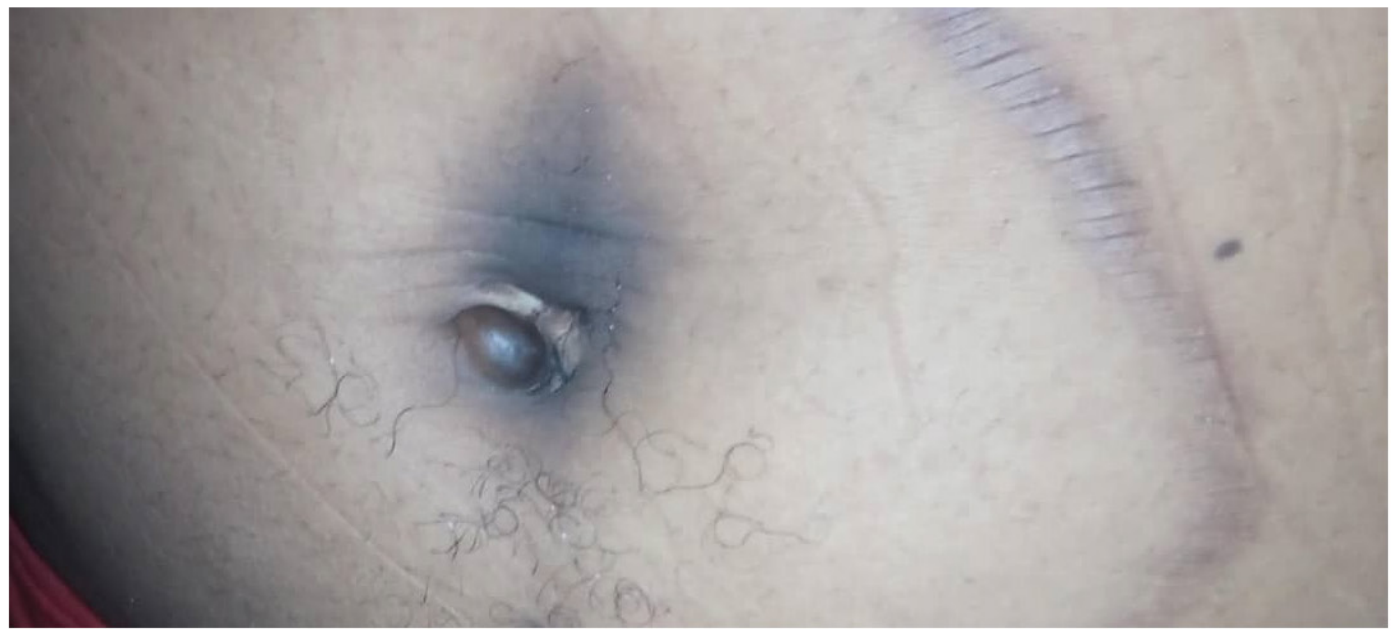

Figure 1: Picture of the endometrotic nodule before excision.

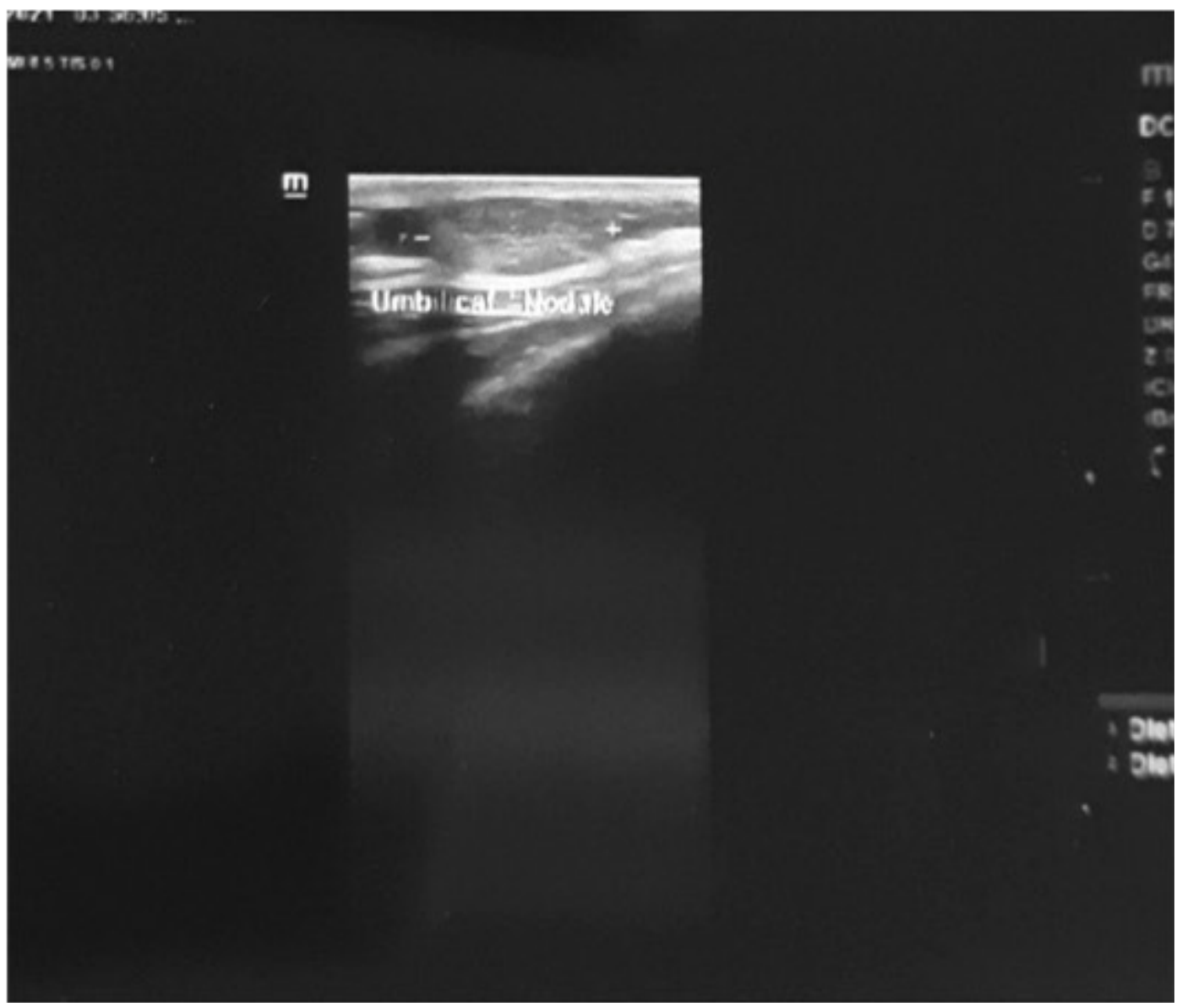

Figure 2: Picture of the ultrasonogram of the umbilical nodule.

diagnosis of a Sister Mary Joseph node was made to rule out a possible endometriotic nodule. An abdominopelvic ultrasound report showed a supraumbilical mass with homogeneous echotexture measuring $2 \mathrm{~cm} \times 3 \mathrm{~cm}$ (Figure 2). The mass was attached to the underlying rectus sheath. Sonodiagnosis of the pelvis revealed an anteverted, non-gravid uterus measuring $5.8 \times 4.5 \mathrm{~mm}$ in length and Anteriorposterior diameter, respectively, in addition the uterus showed a heterogeneous myometrial echo pattern (Figure 3).

No intrauterine or extrauterine gestational sac was noticed. The endometrial cavity was well demonstrated. In addition to the endometriotic nodule, A small solitary isoechoic mass was found in the anterior myometrium measuring $1.2 \mathrm{~cm} \times 2.2 \mathrm{~cm}$ in size suggestive of an intramural uterine myoma seedling. Furthermore, a right-sided unilocular cyst measuring $6.8 \times 6.9 \mathrm{~cm}$, with an acoustic enhancement and a homogenous groundglass echotexture was found suggestive of a possible endometrioma (Figure 3 and Figure 4).

An Excision biopsy of the supraumbilical nodule was performed under general anesthesia to confirm the 


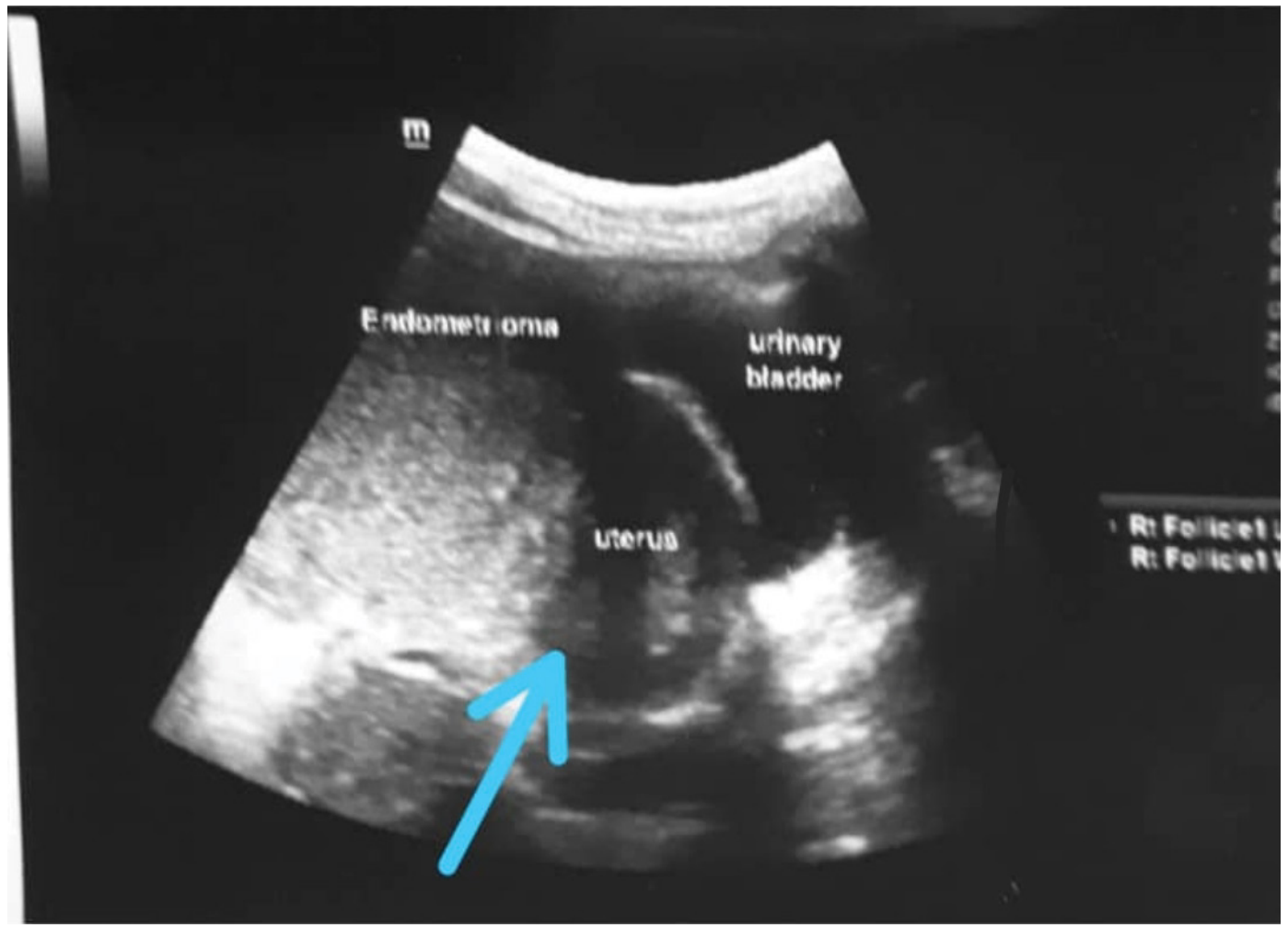

Figure 3: Picture of the abdomino-pelvic sonogram of the endometroma, solitarymyoma (indicated by a blue arrow) and the uterus.

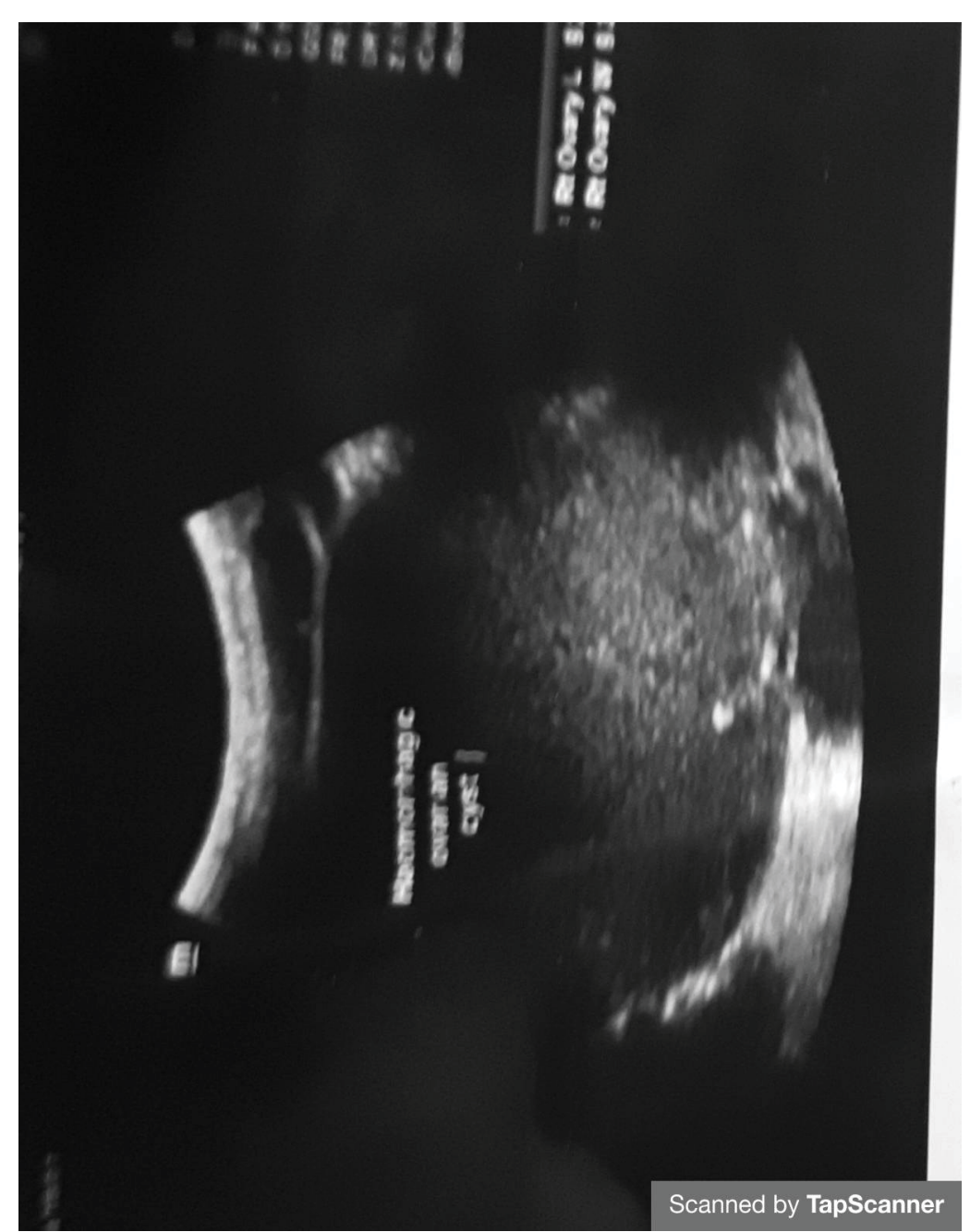

Figure 4: Picture of the zoomed picture of the sonogram of the endometrioma. 
diagnosis of the primary umbilical endometriosis.

The umbilical specimen was sent for a histology review, and the histology examination showed a specimen consisting of 2 pieces of amorphous greyish white tissue both measuring $2.0 \times 1.0 \times 0.5 \mathrm{~cm}$. Cut sections are greyish white. Microscopic examination: Of histologic sections of skin tissue showed islands of unremarkable endometrial glands and stroma, surrounded by hemosiderin-laden macrophages suggestive of primary umbilical endometriosis. Further workup to confirm the endometrioma with Magnetic resonance imaging (MRI) and laparoscopy was requested for the patient, but no further workup was done due to financial limitations.

\section{Discussion}

Endometriosis is a common condition among women of a reproductive age group that negatively impacts their quality of life [9]. Histopathologically, it is defined by the presence of endometrial stroma and glands outside the endometrium [2,9].

While ovarian endometriosis is the commonest form of endometriosis with a prevalence of $88 \%$ of all cases of endometriosis [10], Cutaneous endometriosis is a rare spread of endometriosis found on the anterior abdominal wall, including those that arise from a surgical incision or spontaneously $[5,11]$.

It is identified by the presence of endometrial stroma and glands in the skin [2]. This constitutes less than $1 \%$ of all cases [2].

Cutaneous endometriosis can be primary or secondary. Primary, also known as spontaneous cutaneous endometriosis, is found in the absence of surgery and usually located in the umbilicus, as seen in the index patient [1]. The pathogenesis of endometriosis is still not fully understood with Multiple etiopathogenesis including genetic susceptibility, hormonal, immune and environmental factors has also been discussed [12]. The presence of both endometrioma and extrapelvic (umbilical) endometriosis can be explained by these multifactorial etiopathogenesis [10].

Clinical features of endometriosis include cyclical pain, chronic pelvic pain, dyspareunia, swelling, and even bleeding that correlates with their menstrual cycle $[13,14]$. It is important to note that it is an important cause of infertility [14]. Primary umbilical endometrosis with an endometrioma coexisting with uterine myoma has been reported in some publications [10].

The index patient experienced cyclical pain, dyspareunia, and discoloration in the umbilical swelling around her menstrual cycle. She also had a history of 3 years of infertility. While it is not every patient that presents with all these symptoms, some can be asymptomatic [1]. While cutaneous endometriosis could be suspected clinically, the current gold standard for the diagnosis is histological confirmation [1,8]. Other diagnostically helpful tools include ultrasonography, which is usually the first choice of evaluation, computed tomography, magnetic resonance imaging, and Doppler ultrasonography [14].

The differential diagnosis of cutaneous umbilical endometriosis includes lipoma, keloid, hypertrophic scars, granuloma, hernia, cutaneous metastasis of cancers, among others which were all considered. However, these lesions do not present with cyclical pain and discoloration of the mass as seen in this patient $[1,14]$.

The treatment for cutaneous umbilical endometriosis is generally surgical excision, preferably performed at the end of the menstrual cycle when the lesion is small to clear all the margins to prevent recurrence [1,14]. Our patient had surgical excision of the mass, which was eventually sent for histology but no surgery was done regarding the uterine myoma and endometrioma due to financial constraints.

It can also be treated with hormonal therapy, such as combining oral contraceptive pills and gonadotropinreleasing hormone agonists like danazol, which were not considered in the index patient as there was a history of infertility [1].

Regarding the prognosis, recurrence is the commonest problem encountered with endometriosis following surgical treatment, Primary umbilical endometriosis will recur more than endometrioma [15].

\section{Conclusion}

Since umbilical Endometriosis is a rare finding, a high index of suspicion is needed to make the diagnosis. Any young lady of reproductive-age with a complaint of a swollen painful umbilical nodule without any surgical history should point towards this diagnosis. As a result any patient suspected of primary umbilical endometriosis should undergo detailed history, examinations with investigations in order to confirm and as well as look out for possible endometriosis in other parts of the body.

The management and treatment in poor resource countries can be a daunting task since the procedures needed to confirm and manage the condition is not easily accessible and when available, the price will be a major turnoff for patients.

\section{Acknowledgement}

We hereby acknowledge the patient who gave us the go ahead to write the case report and all the staff of Westcare Hospital Ejigbo, Lagos Nigeria for the roles they played in the management of the patient.

\section{Conflict of Interest}

The authors declare no conflict of interest. 


\section{References}

1. Ade-Ojo IP, Ipinnimo OM (2021) Menstruating primary umbilicus cutaneous endometriosis: $A$ case report and review of the literature. Clin J Obstet Gynecol 4: 69-71.

2. Seung-Hee L, Lew B-L, Sim W-Y (2017) Primary cutaneous endometriosis of umbilicus. Ann Dermatol 29: 621-625.

3. Dimitra C, Koukoura O, Ntavela IG, Chintziou F, Gkorila $G$, et al. (2018) Gastrointestinal and urinary tract endometriosis: A review of the commonest locations of extrapelvic endometriosis. Adv Med.

4. Hyun JL, Park YM, Jee BC, Kim B, Suh CS, et al. (2015) Various anatomic locations of surgically proven endometriosis: A single-center experience. Obstet Gynecol Sci 58: 53-58.

5. Parasar P, Ozcan P, KLT (2018) Endometriosis: Epidemiology. Curr Obs Gynecol Rep 6: 34-41.

6. Bulletti C, Coccia ME, Battistoni S, Borini A (2010) Endometriosis and Infertility. J Assist Reprod Genet 27: 441-447.

7. Johnston Jennifer L, Reid H, Hunter D (2015) Diagnosing endometriosis in primary care. British Journal of General Practice 65: 101-102.

8. Lorenzo C, Sciascia V, Loiaco G, Guida G, larrobino F, et al. (2020) Primary subcutaneous umbilical endometriosis: Case report and review of the literature. Case Rep Surg 2020: 8899618 .
9. Hunt G, Allaire C, Yong PJ, Dunne C (2021) Endometriosis: An update on diagnosis and medical management. BC Med J 63: 158-163.

10. Yunusa DM, Umar UH, Dahiru AMC (2019) Umbilical and ovarian endometriosis coexisting with multiple uterine myomas: A rare case report. Indian J Case Reports 5: 4446.

11. Saliba C, Jaafoury $H$, El Hajj M, Nicolas G, Ahmad HH, et al. (2019) Abdominal wall endometriosis: A Case report. Cureus 11: e4061.

12. Sourial S, Tempest N, Hapangama DK (2014) Theories on the pathogenesis of endometriosis. Int J Reprod Med 2014: 179515.

13. Alimi Y, Iwanaga J, Loukas M, Tubbs RS (2018) The clinical anatomy of endometriosis: A review. Cureus 10: e3361.

14. Matei A-M, Draghici lonescu A-M, Cioplea M, Zurac S, Boda D, et al. (2021) Skin endometriosis: A case report and review of the literature. Exp Ther Med 21: 1-5.

15. Konstantinos N, Ma L, McKinnon B, Mueller MD (2020) Recurrence patterns after surgery in patients with different endometriosis subtypes: A long-term hospital-based cohort study. J Clin Med 9: 496. 